\title{
COMBINED BIMA AND OVRO OBSERVATIONS OF COMET C/1999 S4 (LINEAR)
}

\author{
Michiel R. Hogerheidde, ${ }^{1,2,3}$ Imke de Pater, ${ }^{1}$ Melvyn Wright, ${ }^{1}$ J. R. Forster, ${ }^{4}$ L. E. Snyder,${ }^{5}$ A. Remijan, ${ }^{5}$ \\ L. M. Woodney, ${ }^{6}$ M. F. A'Hearn, ${ }^{7}$ Patrick Palmer, ${ }^{8}$ Y.-J. Kuan, $,{ }^{9}, 10$ H.-C. Huang $,{ }^{9}, 10$ \\ Geoffrey A. Blake, ${ }^{11,12}$ Chunhua Qi ${ }^{11,13}$ Jacqueline Kessler, $^{12}$ and S.-Y. Liu ${ }^{10}$ \\ Received 2001 October 10; accepted 2004 January 2
}

\begin{abstract}
We present results from an observing campaign of the molecular content of the coma of comet C/1999 S4 (LINEAR) carried out jointly with the millimeter arrays of the Berkeley-Illinois-Maryland Association (BIMA) and the Owens Valley Radio Observatory (OVRO). Using the BIMA array in autocorrelation ("single-dish") mode, we detected weak HCN $J=1-0$ emission from comet C/1999 S4 (LINEAR) at $14 \pm 4 \mathrm{mK} \mathrm{km} \mathrm{s}{ }^{-1}$ averaged over the $143^{\prime \prime}$ beam. The 3 days over which emission was detected, 2000 July $21.9-24.2$, immediately precede the reported full breakup of the nucleus of this comet. During this same period, we find an upper limit for HCN $J=1-0$ of 144 mJy beam ${ }^{-1} \mathrm{~km} \mathrm{~s}^{-1}\left(203 \mathrm{mK} \mathrm{km} \mathrm{s}^{-1}\right)$ in the $9^{\prime \prime} \times 12^{\prime \prime}$ synthesized beam of combined observations of BIMA and OVRO in cross-correlation ("imaging") mode. Together with reported values of HCN $J=1-0$ emission in the 28" IRAM $30 \mathrm{~m}$ beam, our data probe the spatial distribution of the HCN emission from radii of 1300 to $19,000 \mathrm{~km}$. Using literature results of HCN excitation in cometary comae, we find that the relative line fluxes in the $12^{\prime \prime} \times 9^{\prime \prime}, 28^{\prime \prime}$, and $143^{\prime \prime}$ beams are consistent with expectations for a nuclear source of $\mathrm{HCN}$ and expansion of the volatile gases and evaporating icy grains following a Haser model.
\end{abstract}

Key words: comets: individual (C/1999 S4 (LINEAR)) — radiative transfer — radio lines: solar system — techniques: interferometric

\section{INTRODUCTION}

The volatile component of comets provides an excellent tracer of the chemical composition present during the formation of the solar system (Mumma, Weissman, \& Stern 1993; Irvine \& Bergin 2000; Irvine et al. 2000). Recent years have seen remarkable progress in this area with the appearances of two especially active comets, C/1996 B2 (Hyakutake) and C/1995 O1 (Hale-Bopp), that passed close to Earth. Observations at (sub-) millimeter wavelengths of these spectacular comets, in addition to numerous less prominent but no less interesting comets over the last decade, indicate that the composition of comets is heterogeneous. This holds not only from comet to comet (e.g., A'Hearn et al. 1995), but also within the same

\footnotetext{
${ }^{1}$ Department of Astronomy, 601 Campbell Hall, University of California at Berkeley, Berkeley, CA 94720.

2 Steward Observatory, University of Arizona, 933 North Cherry Avenue, Tucson, AZ 85721.

${ }^{3}$ Current address: Sterrewacht Leiden, Postbus 9513, NL-2300 RA Leiden, Netherlands; michiel@strw.leidenuniv.nl.

${ }^{4}$ Hat Creek Radio Observatory, 42231 Bidwell Road, Hat Creek, CA 96040.

${ }^{5}$ Department of Astronomy, University of Illinois, 1002 West Green Street, Urbana, IL 61801.

${ }^{6}$ Lowell Observatory, 1400 Mars Hill Road, Flagstaff, AZ 86001.

7 Department of Astronomy, University of Maryland, College Park, MD 20742.

${ }^{8}$ Department of Astronomy and Astrophysics, University of Chicago, 5640 South Ellis Avenue, Chicago, IL 60637.

${ }^{9}$ Department of Earth Sciences, National Taiwan Normal University, 88 Sec. 4, Ting-Chou Road, Taipei 116, Taiwan.

10 Academia Sinica, Institute of Astronomy and Astrophysics, P.O. Box 23-141, Taipei 106, Taiwan.

11 Division of Geological and Planetary Sciences, MS 150-21, California Institute of Technology, 1200 East California Boulevard, Pasadena, CA 91125.

${ }_{12}$ Division of Chemistry and Chemical Engineering, California Institute of Technology, 1200 East California Boulevard, Pasadena, CA 91125.

${ }^{13}$ Harvard-Smithsonian Center for Astrophysics, 60 Garden Street, Cambridge, MA 02138.
}

comet, as was shown with millimeter interferometers that resolve the emission from the coma (e.g., Blake et al. 1999). The latter work illustrated what high spatial resolution observations can show about cometary composition and evaporation processes.

In the summer of 2000, the comet C/1999 S4 (comet LINEAR from here on) passed close to the Sun $(0.765 \mathrm{AU})$ and 0.374 AU from Earth (Durig, Ticha, \& Tichy 1999). Its orbit identified it as a dynamically young comet, and it showed a high level of activity at large heliocentric distance. As is common in dynamically young comets, the increase in its activity lagged significantly behind what was expected after its discovery, reaching only a medium level of activity at perihelion passage. It generated some excitement when, at closest approach to the Sun, the nucleus of the comet disintegrated and the residual material faded away in a few days, as first reported by Kidger (2000). The comet's characteristics and its breakup are discussed in detail by Bockelée-Morvan et al. (2001), Farnham et al. (2001), Lisse et al. (2001), Mäkinen et al. (2001), Mumma et al. (2001), Weaver et al. (2001), Bonev et al. (2002), Altenhoff et al. (2002), Tozzi \& Licandro (2002), Schulz \& Stüwe (2002), Cochran \& Cochran (2001), Jockers et al. (2001), Rodríguez et al. (2002), Kidger (2002), and Magee-Sauer et al. (2001). Of most relevance to our discussion here are the conclusion that the comet's nucleus may already have broken up several weeks before it fully disintegrated in late July (July 23-24), the molecular line observations reported by Bockelée-Morvan et al. including the $\mathrm{HCN}$ production rate, and the infrared HCN line observations of Magee-Sauer et al.

This paper describes the results of an observing campaign of the volatile composition of comet LINEAR, carried out jointly by the millimeter arrays at Hat Creek (operated by the Berkeley-Illinois-Maryland Association) and Owens Valley (operated by the California Institute of Technology), both in California ( $(2)$. We present a weak detection of $\mathrm{HCN} J=1-0$ in autocorrelation mode and upper limits on the emission in 
TABLE 1

OBSERVATIONS

\begin{tabular}{|c|c|c|c|c|c|}
\hline $\begin{array}{c}\text { OVRO } \\
\text { Cross-Correlation } \\
\text { (UT 2000 Jul) }\end{array}$ & $\begin{array}{c}\text { BIMA } \\
\text { Cross-Correrlation } \\
\text { (UT 2000 Jul) }\end{array}$ & $\begin{array}{c}\text { BIMA } \\
\text { Autocorrelation } \\
\text { (UT 2000 Jul) }\end{array}$ & $\begin{array}{c}\text { JPL Orbit } \\
\text { Solution No. }\end{array}$ & $\begin{array}{c}\text { Gain } \\
\text { Calibrator }^{\mathrm{a}}\end{array}$ & $\begin{array}{l}\text { Calibrator } \\
\text { Flux } \\
(\mathrm{Jy})\end{array}$ \\
\hline $18.47-19.15 \ldots \ldots \ldots$ & $\ldots$ & $\ldots$ & 62 & $0716+714$ & 3.0 \\
\hline $19.47-19.88 \ldots \ldots \ldots$ & $\ldots$ & $\ldots$ & 68 & $0716+714$ & 3.0 \\
\hline $19.95-19.99 \ldots \ldots \ldots$ & $\ldots$ & $\ldots$ & 68 & $0716+714$ & 3.0 \\
\hline $20.50-20.93 \ldots \ldots \ldots$ & $20.45-20.73$ & $\ldots$ & 77 & $0716+714$ & 3.0 \\
\hline $21.55-22.13 \ldots \ldots \ldots$ & $21.69-21.82$ & $21.85-22.13$ & 77 & $0923+392$ & 4.5 \\
\hline $22.59-23.14 \ldots \ldots \ldots$ & $22.63-22.92$ & $22.95-23.12$ & 78 & $0923+392$ & 4.5 \\
\hline $23.64-24.08 \ldots \ldots \ldots$. & $23.65-23.92$ & $23.95-24.16$ & 82 & $0923+392$ & 4.5 \\
\hline $24.70-25.12 \ldots \ldots \ldots$ & $24.67-24.92$ & $24.95-25.12$ & 82 & $0923+392$ & 4.5 \\
\hline $25.72-26.16 \ldots \ldots \ldots$ & $25.69-25.94$ & $25.99-26.30$ & 83 & $0923+391$ & 4.5 \\
\hline $26.76-27.19 \ldots \ldots \ldots$ & $26.70-26.89$ & $26.92-27.17$ & 87 & $1156+295$ & 1.2 \\
\hline
\end{tabular}

${ }^{\text {a }}$ Source designation following B1950-based OVRO calibrator list (http://www.ovro.caltech.edu/mm/preobs/guide.html).

cross-correlation mode and place limits on the spatial extent of the emission ( $(3)$. Using models of $\mathrm{HCN}$ excitation in comets from Crovisier (1987) (see also Bockelée-Morvan et al. 1984), we investigate whether the spatial extent matches expectations (§ 4). Our conclusions are summarized in $\S 5$. The Appendix discusses the methods used to combine data from the two millimeter arrays.

\section{OBSERVATIONS}

Observations of comet LINEAR were obtained between 2000 July 17 and 2000 July 27 at the millimeter interferometers of the Berkeley-Illinois-Maryland Association (BIMA) ${ }^{14}$ at Hat Creek, California ( $121^{\circ} 28^{\prime} 18^{\prime \prime} 49$ west, $40^{\circ} 49^{\prime} 2^{\prime \prime} .5$ north; altitude $1043 \mathrm{~m}$ ), and the Caltech Millimeter Array at the Owens Valley Radio Observatory (OVRO $)^{15}$ near Big Pine, California $\left(118^{\circ} 16^{\prime} 55^{\prime \prime}\right.$.92 west, $37^{\circ} 14^{\prime} 2^{\prime \prime}$.04 north; altitude $\left.1222 \mathrm{~m}\right)$. The six $10 \mathrm{~m}$ antennas of the OVRO array were in "compact" configuration; the ten $6 \mathrm{~m}$ antennas of the BIMA array were in "C" configuration. Both configurations result in comparable projected baselines: $2-20 \mathrm{k} \lambda$ at BIMA and $3-15 \mathrm{k} \lambda$ at OVRO. Since these were summertime observations, when atmospheric stability can be poor for millimeter interferometry, these compact configurations were adopted to avoid decorrelation by atmospheric phase fluctuations on long baselines.

OVRO observed the comet in cross-correlation mode from rise in the early morning local time until set in the late afternoon local time. BIMA operated in cross-correlation mode from rise until midmorning local time. After midmorning, the phase stability at Hat Creek deteriorated significantly during the observing period, and the observing mode was switched to autocorrelation. This observing mode does not suffer from phase decorrelation but has a large beam dilution in the 143" full width at half-maximum (FWHM) primary beam of the $6 \mathrm{~m}$ antennas. The phase stability at Owens Valley, approximately $500 \mathrm{~km}$ southeast of Hat Creek and on the opposite side of the Sierra Nevada mountain range, was significantly better than Hat Creek, and successful cross-correlation data were obtained throughout each day. In addition, the digital correlator at OVRO cannot operate in autocorrelation mode, which fortunately was not a limitation.

\footnotetext{
${ }^{14}$ The BIMA Array is operated by the Berkeley-Illinois-Maryland Association under funding from the National Science Foundation.

${ }^{15}$ The Owens Valley Millimeter Array is operated by the California Institute of Technology under funding from the US National Science Foundation (AST 96-13717).
}

Receivers and digital correlators of both instruments were configured to record the lines of $\mathrm{HCN} J=1-0$ $(88.6318470 \mathrm{GHz})$ and $\mathrm{HCO}^{+} J=1-0(89.1885230 \mathrm{GHz})$. At OVRO, each of the $\mathrm{HCN}$ and $\mathrm{HCO}^{+}$line frequencies were recorded in a single band of 64 channels with a resolution of $125 \mathrm{kHz}\left(0.42 \mathrm{~km} \mathrm{~s}^{-1}\right)$. At BIMA, the lines were recorded in two bands per line, centered on the line frequency, with 128 and 256 channels each and with respective channels widths of 391 and $98 \mathrm{kHz}\left(1.3\right.$ and $\left.0.33 \mathrm{~km} \mathrm{~s}^{-1}\right)$. The autocorrelation mode of the BIMA correlator offers twice the number of channels at half the width for each band, giving velocity resolutions of 0.65 and $0.17 \mathrm{~km} \mathrm{~s}^{-1}$.

Great care was taken to ensure that both arrays were using the same comet ephemeris at all times. On each observing day, the most recent orbit solution from the Jet Propulsion Laboratory Solar System Dynamics Group ${ }^{16}$ was used (Table 1). Changes of the predicted comet's position between subsequent orbit solutions were less than a few arcseconds, smaller than the synthesized beam of $\sim 12^{\prime \prime}$ and much smaller than the primary beam of $143^{\prime \prime}$ for the BIMA autocorrelation observations. The small day-to-day uncertainty in the comet's position therefore has no appreciable effect on the data. Because OVRO and BIMA use different coordinate systems (B1950.0 vs. J2000, respectively) and use, respectively, geocentric and topocentric representations for the coordinates of solar system objects, much effort was spent to ensure that both instruments actually pointed at the same position on the sky. Note that the parallax of the comet at a geocentric distance of $0.3 \mathrm{AU}$ amounts to $\sim 2^{\prime \prime}$ over the distance between the two arrays. The topocentric velocity of the comet was also Doppler tracked at both sites, keeping the cometocentric velocity centered at $0 \mathrm{~km} \mathrm{~s}^{-1}$ in the spectral bands.

The complex gains of cross-correlation data were calibrated separately for the BIMA and OVRO data, although the same flux and phase calibrators were used for both data. Depending on the position on the sky of the comet, the quasars $0716+714$, $0923+392$, and $1156+295$ were used, with respective fluxes of $3.0,1.2$, and $1.2 \mathrm{Jy}$ as determined from contemporaneous measurements of planets at OVRO (Table 1). Using the same calibrator at each observatory ensures that the flux scaling of both data sets is identical. The OVRO data were calibrated using the MMA package specific to Owens Valley (Scoville et al. 1993); the BIMA data were calibrated using the

\footnotetext{
${ }^{16}$ Available at http://ssd.jpl.nasa.gov/horizons.html.
} 
MIRIAD software package (Sault, Teuben, \& Wright 1995). The OVRO data, originally stored in FITS format, were subsequently converted to MIRIAD format, and both crosscorrelation data sets were added together after regridding to a common spectral resolution. The Appendix describes the data combination procedure in more detail, including a test case validating the process. The combined data were then inverted and imaged using MIRIAD.

The autocorrelation observations were corrected for atmospheric opacity and fluctuations by switching to an "off" position at $+20^{\prime}$ in right ascension every $30 \mathrm{~s}$. The data were recorded on the main beam brightness temperature scale in $\mathrm{K}$; subsequent observations of the Galactic $\mathrm{SiO}$ maser $\mathrm{R}$ Leo in cross- and autocorrelation modes confirmed the flux scaling. A number of bad channels were flagged because of spikes, and second- to fourth-order polynomial baselines were subtracted from the autocorrelation spectra for the individual antennas. Finally, the spectra from the individual antennas were added to yield a single autocorrelation spectrum for each spectral band. All processing of the autocorrelation data was carried out within MIRIAD.

\section{RESULTS}

\subsection{Autocorrelation Spectra}

The autocorrelation spectra of $\mathrm{HCN} J=1-0$ (Fig. 1) and $\mathrm{HCO}^{+} J=1-0$ were inspected using the average data of single, individual days and averaging over 2,3 , and 6 consecutive days. In HCN, a weak signal was detected in 2 and 3 day averages between 2000 July 21.85 and 2000 July 24.16. These are the 3 days leading up to the disruption of the nucleus between 2000 July 24.9 and 25.9 (Kidger 2000), and they coincide with a reported brightening at visible wavelengths, likely indicating a minor outburst event. The 3 day average main beam brightness temperature of HCN $J=1-0$ is $14 \pm 4 \mathrm{mK} \mathrm{km} \mathrm{s}^{-1}$ in the $143^{\prime \prime}$ FWHM beam of the $6 \mathrm{~m}$ dishes. The emission is centered around a velocity of $-1 \mathrm{~km}$ $\mathrm{s}^{-1}$, close to the expected velocity of the comet (nominally at zero), and has a width of $\sim 2 \mathrm{~km} \mathrm{~s}^{-1}$ FWHM. The latter value is uncertain because of the low signal-to-noise ratio $(\mathrm{S} / \mathrm{N})$ of the emission; the hyperfine components at +4 and $-7 \mathrm{~km} \mathrm{~s}^{-1}$ were likewise below the noise level. The reported intensity is a total for all three components, obtained by multiplying the integrated intensity of the single detected component by the expected relative intensities in thermodynamic equilibrium: $0.2+1.0+0.6=1.8$. Although the overall signal-to-noise of the detection is low, the 3 day averages leading up to (2000 July $21.85-24.16 ; 14 \pm 4 \mathrm{mK} \mathrm{km} \mathrm{s}^{-1}$ ) and following (2000 July $24.95-27.17 ;<10 \mathrm{mK} \mathrm{km} \mathrm{s}{ }^{-1}, 2 \sigma$ upper limit) the comet's final breakup around 2000 July $23-24$ clearly show a dearth of HCN $J=1-0$ emission. The decrease in HCN $J=$ 3-2 emission observed with the IRAM $30 \mathrm{~m}$ telescope by Bockelée-Morvan et al. (2001) after 2000 July 23.8 supports our detected falloff of HCN. No emission was detected in any

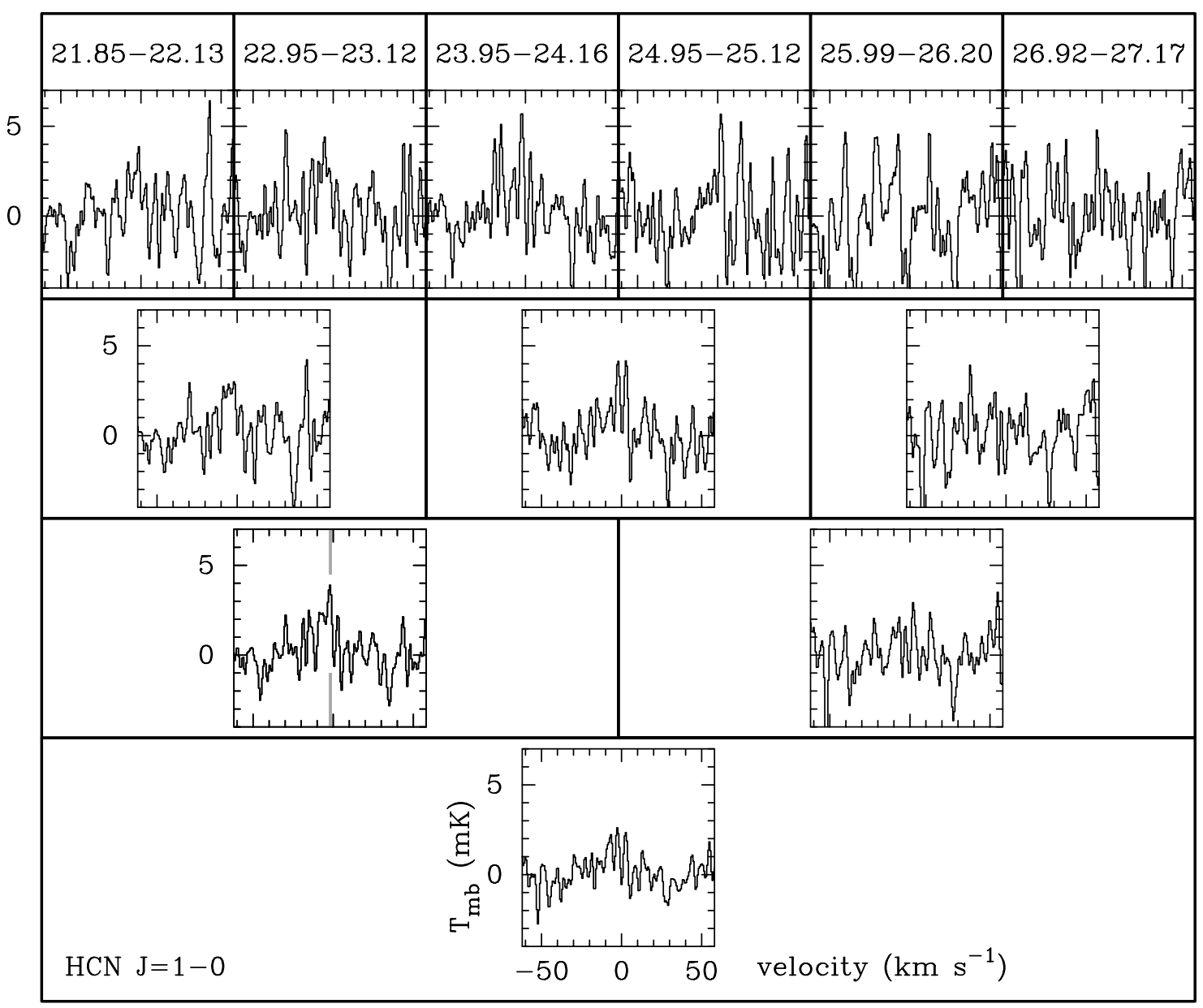

FIG. 1.-Autocorrelation spectra obtained in the 143" BIMA primary beam. The top row shows single day averages; the subsequent rows show the 2 day, 3 day, and 6 day averages. Emission of $\mathrm{HCN} J=1-0$ is detected at $\sim 4 \sigma$ averaged over 2001 July 21.65-24.16, indicated by the gray bar in the corresponding panel. 
of the $\mathrm{HCO}^{+}$spectra, to a $2 \sigma$ upper limit of $4 \mathrm{mK} \mathrm{km} \mathrm{s}^{-1}$ in 3 day averages.

\subsection{Cross-Correlation Images}

The cross-correlation data were inspected in a similar way as the autocorrelation data. The data were integrated over a single day, over multiple consecutive days, and over $4 \mathrm{hr}$ time intervals to search for transient features. This was done for the data from the two arrays individually as well as for the combined data set. In all cases, the emission was integrated over $2 \mathrm{~km} \mathrm{~s}^{-1}$ around a cometocentric velocity that was varied from -10 to $+10 \mathrm{~km} \mathrm{~s}^{-1}$ to account for possible small tracking offsets in the comet's velocity. The reported flux (limits) refer to the sum of the hyperfine components, added with the appropriate weights $(1: 5: 3)$.

None of the images obtained in this way revealed any positive detection of $\mathrm{HCN} J=1-0$ or $\mathrm{HCO}^{+} J=1-0$ emission. Figure 2 illustrates this for $\mathrm{HCN} J=1-0$ at an adopted cometocentric velocity of $0 \mathrm{~km} \mathrm{~s}^{-1}$ and includes all OVRO and BIMA data. Using the 3 day average over 2000 July 21.8524.16, over which emission was detected in autocorrelation mode, an upper limit to the HCN $J=1-0$ emission is found of 144 mJy beam ${ }^{-1} \mathrm{~km} \mathrm{~s}^{-1}(2 \sigma)$ in the $12^{\prime \prime} \times 9^{\prime \prime}$ synthesized (naturally weighted) beam, corresponding to $203 \mathrm{mK} \mathrm{km} \mathrm{s}{ }^{-1}$. The corresponding values for $\mathrm{HCO}^{+} J=1-0$ are smaller, at $80 \mathrm{mJy}^{\text {beam }}{ }^{-1} \mathrm{~km} \mathrm{~s}^{-1}$ and $117 \mathrm{mK} \mathrm{km} \mathrm{s}^{-1}$, because $\mathrm{HCO}^{+}$has no hyperfine splitting to sum over.

\section{COMPARISON TO MODEL CALCULATIONS}

In $\S 3$, we showed that $\mathrm{HCN} J=1-0$ emission was detected at low $\mathrm{S} / \mathrm{N}$ in autocorrelation mode but not in crosscorrelation mode. If the emission is unresolved $\left(<10^{\prime \prime}\right)$, the beam-averaged value of $14 \pm 4 \mathrm{mK} \mathrm{km} \mathrm{s}^{-1}$ detected in the $143^{\prime \prime}$ primary beam corresponds to an intensity of $2.7 \pm 0.8 \mathrm{~K}$ $\mathrm{km} \mathrm{s}^{-1}$ in the $12^{\prime \prime} \times 9^{\prime \prime}$ synthesized beam of the cross-correlation data. Since we obtained an upper limit of $203 \mathrm{mK} \mathrm{km}$ $\mathrm{s}^{-1}$, it follows that the emission is significantly resolved out in the interferometer beam. Alternative explanations for reduced flux in the cross-correlation data are decorrelation due to uncorrected phase variations and positional uncertainties of the comet larger than the synthesized beam. These processes are unlikely to affect the data: the measured decorrelation on the phase calibrator is typically less than $20 \%$, and as mentioned in $\S 2$, the position of the nucleus from day to day was known to within a few arcseconds, which is much smaller than the synthesized beam.

Extended emission is expected from molecules in the expanding coma. For a parent species like $\mathrm{HCN}$, the density in the coma follows a Haser distribution

$$
n(r)=\frac{Q}{4 \pi V_{0} r^{2}} \exp \left(-\frac{r-r_{n}}{r_{\lambda}}\right),
$$

where $n$ is the number density of molecules, $Q$ the production rate of that molecule by the nucleus, $V_{0}$ is the expansion

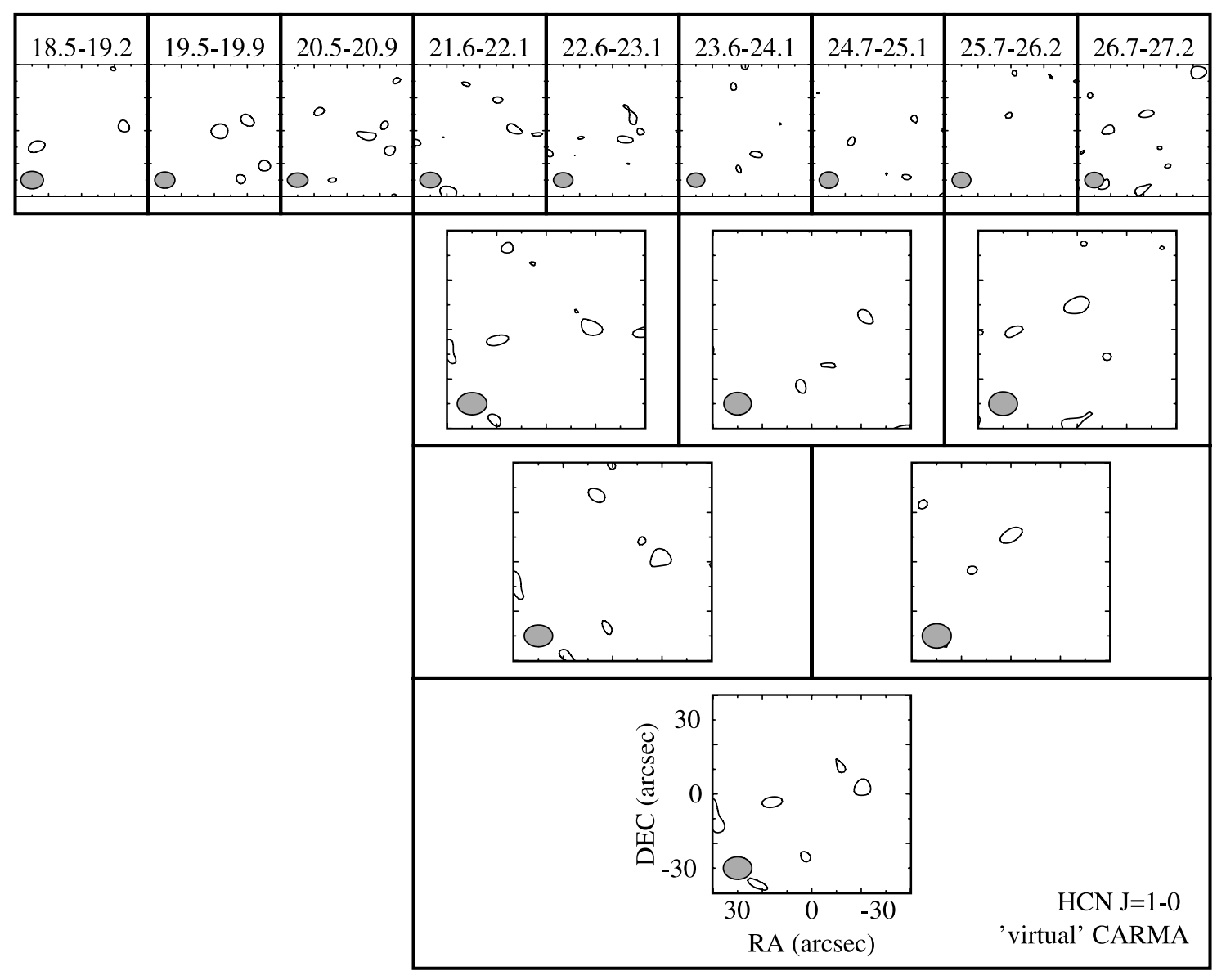

FIG. 2. Cross-correlation images of the combined BIMA and OVRO data centered on a velocity of $0 \mathrm{~km} \mathrm{~s}^{-1}$, with $2 \sigma$ contours $\left(1 \sigma=42 \mathrm{mJy}\right.$ beam $^{-1} \mathrm{~km} \mathrm{~s}^{-1}$

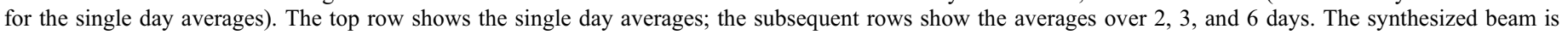
indicated in the lower left corner of each panel. 
velocity in the coma, $r_{n}$ is the size of the nucleus, and $r_{\lambda}$ is the characteristic photodissociation scale length. BockeléeMorvan et al. (2001) quote $Q(\mathrm{HCN})=3 \times 10^{25} \mathrm{~s}^{-1}$; we adopt $V_{0}=0.9 \mathrm{~km} \mathrm{~s}^{-1}$ and $r_{\lambda}=V_{0} / \beta=3.7 \times 10^{4} \mathrm{~km}$, where $\beta=2.45 \times 10^{-5} \mathrm{~s}^{-1}$ is the $\mathrm{HCN}$ photodissociation rate for the solar spectrum at the time of the observations (D. BockeléeMorvan 2001, private communication).

Is a distribution of $\mathrm{HCN}$ following equation (1) consistent with our detection in the $143^{\prime \prime}$ autocorrelation beam and our upper limit in the $12^{\prime \prime} \times 9^{\prime \prime}$ cross-correlation beam, or is an additional source of $\mathrm{HCN}$ required, distributed over $>10^{\prime \prime}$, e.g., evaporating icy fragments that have broken off the nucleus? Fragments exceeding $\sim 1 \mathrm{~m}$, nonuniformly illuminated by the Sun, are expected to undergo nongravitational forces and accelerate away from the inner $\left(\sim 10^{\prime \prime}\right)$ coma (e.g., Desvoivres et al. 1999). Our data uniquely probe the spatial distribution of the $\mathrm{HCN} J=1-0$ emission. BockeléeMorvan (2001, private communication) report $\mathrm{HCN} J=1-0$ emission in the $28^{\prime \prime}$ IRAM $30 \mathrm{~m}$ beam of $93 \pm 13 \mathrm{mK} \mathrm{km} \mathrm{s}^{-1}$, averaged over 2000 July 21-23; we include this data point in our analysis. We do not consider the HCN $J=3-2$ emission reported by Bockelée-Morvan et al. (2001) here, because we limit our discussion to the $J=1-0$ transition.

The emission distribution of $\mathrm{HCN} J=1-0$ depends on the spatial distribution of the molecules following equation (1) and on their excitation as function of position. The latter is given by the balance of (de-)excitation through collisions with water and electrons, excitation into vibrationally excited levels through absorption of solar infrared photons, and de-excitation through spontaneous emission. Bockelée-Morvan et al. (1984) and Crovisier (1987) discuss the excitation of HCN in comets in detail. Instead of carrying out a full excitation calculation, we use the HCN level populations modeled by Crovisier (1987). This paper presents three cases: water production rate $Q\left(\mathrm{H}_{2} \mathrm{O}\right)=2 \times 10^{29} \mathrm{~s}^{-1}$ and heliocentric distance $r_{h}=1.0 \mathrm{AU}$ (model 1),$Q\left(\mathrm{H}_{2} \mathrm{O}\right)=2 \times 10^{28} \mathrm{~s}^{-1}$ and $r_{h}=1.6 \mathrm{AU}$ (model 2), and $Q\left(\mathrm{H}_{2} \mathrm{O}\right)=1 \times 10^{30} \mathrm{~s}^{-1}$ and $r_{h}=0.8$ AU (model 3). None of the parameters of these models are exactly identical to those for comet LINEAR $\left[Q\left(\mathrm{H}_{2} \mathrm{O}\right)=3 \times 10^{28} \mathrm{~s}^{-1}\right.$, BockeléeMorvan et al. 2001; and $\left.r_{h}=0.76 \mathrm{AU}\right]$, but we show that the relative line intensities in particular in the $12^{\prime \prime} \times 9^{\prime \prime}, 28^{\prime \prime}$, and $143^{\prime \prime}$ beams are comparatively insensitive to the model details and are largely determined by the underlying column density distribution. Although the exact values of the relative line intensities in the three beams depend on the details of the excitation distribution in the coma that results from the balance between fluorescent and collisional processes, values within the same range are found in the extreme cases in which either of these processes dominate. The models described above can therefore be used to test whether our data agree with a Haser distribution for the column density.

Using a Haser distribution following equation (1) with the parameters listed above and the $J=0$ and $J=1 \mathrm{HCN}$ level populations of the three models from Crovisier (1987), we calculate the expected HCN $J=1-0$ emission distributions at a distance of $0.374 \mathrm{AU}$ from Earth. We convolve the emission distributions with Gaussians of $143^{\prime \prime}$ and $28^{\prime \prime}$ FWHM to get the main beam intensities in these beams. For the crosscorrelation data, we construct synthetic visibilities based on the predicted emission distribution and the actual antenna positions of the data. This explicitly takes into account the spatial filtering of the interferometers and their insensitivity to large-scale structure, as well as the data combination procedure outlined in the Appendix. We process the synthetic visibilities in the same way as the data to obtained integrated intensity images. We find that no more than $10 \%-30 \%$ of the flux is resolved out by the interferometer.

Table 2 lists the observational results in the $143^{\prime \prime}, 28^{\prime \prime}$, and $12^{\prime \prime} \times 9^{\prime \prime}$ beams, alongside the predicted intensities in these beams for the three models. In terms of absolute intensities, the observed values are within a factor of 2 of the model results, indicating a general agreement between the models and the observations. Table 2 also lists the observed and modeled intensities normalized to the value in the $28^{\prime \prime}$ beam: $F_{\text {auto }} / F_{28}$ and $F_{\text {cross }} / F_{28}$. For the three models, $F_{\text {auto }} / F_{28}$ ranges from 0.21 to 0.28 . Our observed value, $0.15 \pm 0.05$, is

TABLE 2

HCN $J=1-0$ Intensities: Observations and Models

\begin{tabular}{|c|c|c|c|}
\hline & $\begin{array}{c}\text { BIMA } \\
\text { Autocorrelation } \\
143^{\prime \prime}\end{array}$ & $\begin{array}{c}\text { IRAM } 30 \mathrm{~m} \\
28^{\prime \prime}\end{array}$ & $\begin{array}{c}\text { BIMA+OVRO } \\
\text { Cross-correlation } \\
12^{\prime \prime} \times 9^{\prime \prime}\end{array}$ \\
\hline \multicolumn{4}{|c|}{ Integrated intensities, in $\mathrm{mK} \mathrm{km} \mathrm{s}^{-1}$} \\
\hline Observed ......................... & $14 \pm 4$ & $93 \pm 13^{\mathrm{a}}$ & $<203$ \\
\hline Model $1^{\mathrm{b}}$.. & 12.4 & 44.4 & 110.0 \\
\hline Model $2^{\mathrm{c}}$. & 22.7 & 110.0 & 172.8 \\
\hline Model $3^{\mathrm{d}}$. & 5.96 & 24.6 & 89.9 \\
\hline \multicolumn{4}{|c|}{ Integrated intensities, normalized to $28^{\prime \prime}$ beam } \\
\hline Observed . & $0.15 \pm 0.05$ & $\equiv 1$ & $<2.5$ \\
\hline Model 1 ............................ & 0.28 & $\equiv 1$ & 2.15 \\
\hline Model $2 \ldots \ldots \ldots \ldots \ldots \ldots \ldots \ldots$ & 0.21 & $\equiv 1$ & 1.57 \\
\hline Model $3 \ldots \ldots \ldots \ldots$ & 0.24 & $\equiv 1$ & 3.65 \\
\hline
\end{tabular}

NoTE.-Observed line intensities and upper limit are averages over 2000 July 21-23. $Q(\mathrm{HCN})=3 \times 10^{25} \mathrm{~s}^{-1}$ has been adopted for the calculations, following Bockelée-Morvan et al. (2001).

${ }^{\text {a }}$ D. Bockelée-Morvan 2000, private communication.

${ }^{\text {b }} Q\left(\mathrm{H}_{2} \mathrm{O}\right)=2 \times 10^{29} \mathrm{~s}^{-1}, r_{h}=1.0 \mathrm{AU}$, from Crovisier (1987), Fig. $2 c$.

c $Q\left(\mathrm{H}_{2} \mathrm{O}\right)=2 \times 10^{28} \mathrm{~s}^{-1}, r_{h}=1.6 \mathrm{AU}$, from Crovisier (1987), Fig. $3 c$.

d $Q\left(\mathrm{H}_{2} \mathrm{O}\right)=1 \times 10^{30} \mathrm{~s}^{-1}, r_{h}=0.8 \mathrm{AU}$, from Crovisier (1987), Fig. $4 c$. 
consistent with the models at the $\sim 1 \sigma$ level. Given the possibility of calibration differences between the IRAM $30 \mathrm{~m}$ data and the BIMA data, we conclude that this indicates that the line emission over $143^{\prime \prime}$ is consistent with the expectation of a Haser distribution when compared to a $28^{\prime \prime}$ region. Bockelée-Morvan et al. (2001) estimate that only fragments with sizes $\gtrsim 1 \mathrm{~m}$ are large enough to survive outside the inner few thousand $\mathrm{km}$ of the coma. Our $143^{\prime \prime}$ result shows that such fragments, if they exist, do not carry a significant contribution of the volatile material $(\$ 50 \%)$. This is consistent with the results of Magee-Sauer et al. (2001), who report that spatially resolved infrared $\mathrm{HCN}$ line observations are consistent with a nuclear source, and of Bockelée-Morvan et al. (2001), who conclude that the evolution of the $\mathrm{HCN}$ production rate suggests that $\mathrm{HCN}$ is mainly released by small icy fragments.

The ratios $F_{\text {cross }} / F_{28}$ of the three models show a larger range, $1.57-3.65$, compared to $F_{\text {auto }} / F_{28}$. This indicates that on these smaller scales, excitation differences exist depending on the heliocentric distance and evaporation rate (which affects the density of collision partners). Our nondetection of emission in the cross-correlation beam yields a strict upper limit, $F_{\text {cross }} / F_{28}<2.5$. This is consistent with the range predicted by the models, especially when taking into account possible decorrelation of our cross-correlation data of up to $20 \%$ (see above) and, again, possible calibration differences between the IRAM $30 \mathrm{~m}$ and the OVRO+BIMA data. In other words, the nondetection in our combined cross-correlation data is expected for a Haser model HCN distribution. This indicates that to obtain high spatial resolution (sub-) millimeter observations of moderately active comets such as LINEAR, higher sensitivity is required. The Combined Array for Research in Millimeter Astronomy (CARMA), planned to be operational in 2005, will provide the increase in sensitivity required for a marginal detection of a comet like LINEAR; with the advent of the Atacama Large Millimeter Array (ALMA), such objects will be easily accessible. Needless to say, detailed excitation calculations along the lines of Bockelée-Morvan et al. (1984), Crovisier (1987), and Biver et al. (1999) will be needed to interpret these future observations.

\section{CONCLUSIONS}

1. We detected $\mathrm{HCN} J=1-0$ emission in autocorrelation mode with the BIMA array at $14 \pm 4 \mathrm{mK} \mathrm{km} \mathrm{s}^{-1}$ in the $143^{\prime \prime}$ beam and find a $2 \sigma$ upper limit of $144 \mathrm{mJy}$ beam ${ }^{-1} \mathrm{~km} \mathrm{~s}^{-1}$ (203 $\mathrm{mK} \mathrm{km} \mathrm{s}^{-1}$ ) in the $12^{\prime \prime} \times 9^{\prime \prime}$ synthesized beam of combined cross-correlation data from OVRO and BIMA. Only upper limits are obtained for $\mathrm{HCO}^{+}$of less than $4 \mathrm{mK} \mathrm{km} \mathrm{s}^{-1}$ in autocorrelation and less than $80 \mathrm{mJy} \mathrm{beam}^{-1} \mathrm{~km} \mathrm{~s}^{-1}$ in crosscorrelation. HCN emission was detected in the 3 days preceding (2000 July 21.9-24.2) the breakup of the comet's nucleus around 2000 July 23-24; no emission in $\mathrm{HCN}$ or $\mathrm{HCO}^{+}$was detected after the breakup.

2. Compared to models of $\mathrm{HCN}$ excitation in cometary comae, we find that the relative and absolute integrated intensities in the $143^{\prime \prime}, 28^{\prime \prime}$, and $12^{\prime \prime} \times 9^{\prime \prime}$ beams are consistent with expectations. This indicates a nuclear source for $\mathrm{HCN}$, and no significant contribution from a spread-out $(>1300 \mathrm{~km})$ distribution of evaporating icy fragments. Fragments exceeding $1 \mathrm{~m}$ in size are thought to be able to resist evaporation over such distances, and our data limit their contribution to the volatiles to $\lesssim 50 \%$ during the days of 2000 July $21-23$ immediately preceding the full disruption of comet LINEAR.

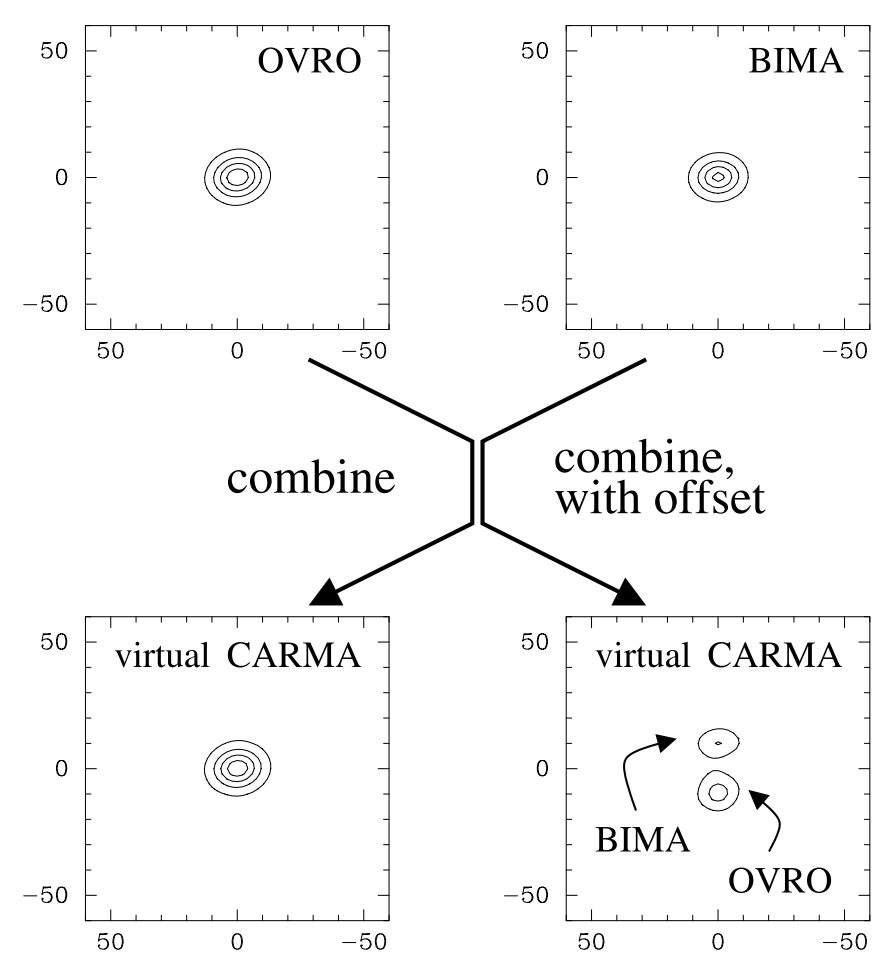

FIG. 3.- Top two panels show the model image as observed by OVRO (left) and BIMA (right). After combination, the "virtual CARMA" result is shown at the lower left. By introducing an offset in the source position of $+10^{\prime \prime}$ for the BIMA model and $-10^{\prime \prime}$ for the OVRO model, the resulting image in the combined data (lower right) is split. This panel shows that the data combination procedure preserves the data from both arrays.

3. We showed that data obtained at the BIMA and OVRO interferometers can be successfully combined, creating a "virtual" CARMA array that increases the signal-to-noise by approximately a factor of $\sqrt{2}$ because of the increased collecting array. While, for these observations, the array configurations were chosen to provide overlapping, short $u v$ spacings because of atmospheric phase stability concerns, virtual CARMA observations may offer complementary array configurations, increasing imaging capabilities. These, however, fall short of what a truly combined array with baselines between the individual BIMA and OVRO antennas can offer (a combined CARMA array is foreseen to be operational by 2005). With the arrival of ALMA, spatially resolved comet observations at (sub-) millimeter wavelengths will become increasingly accessible.

We are indebted to the staffs of the Owens Valley and Hat Creek Radio Observatories for providing excellent support during the observations. Wilson Hoffman and Steve Scott are especially thanked for creating and maintaining the software required to track fast-moving targets such as this comet. The research of M. R. H. in Berkeley was supported by the Miller Institute for Basic Research in Science. This work was partially funded by NASA NAG 5-4292, NAG 5-4080, NAG 5-8708, and NGT 5-0083, NSF AST 96-13998, AST 96-13999, AST 96-13716, AST 96-15608, and AST 99-81363, Taiwanese grants NSC 86-2112-M-003-T and 89-2112-M-003-004, and the Universities of Illinois, Maryland, and California, Berkeley. We greatly thank the referee, D. Bockelée-Morvan, 
for providing constructive criticism of our paper and pointing out ways in which to significantly improve our analysis.

\section{APPENDIX}

\section{COMBINING DATA FROM DIFFERENT INTERFEROMETER ARRAYS}

The results presented in this paper include images that are constructed based on data obtained simultaneously at two different millimeter interferometers, BIMA and OVRO. The process of combining such data contains a number of steps in which special care needs to be taken to ensure that the resulting images correctly represent the data obtained at the individual arrays. This appendix describes in detail the data combination process using MIRIAD and presents simulations that validate the procedure (Fig. 3).

As a first step, even before the data are taken, one needs to ensure that both arrays are using the same source position as the phase-tracking system and that the receivers and correlators are configured to record the same line frequency using the same systemic velocity of the object at all times. Especially for a moving target like a comet, these requirements are not trivial when dealing with two separate observatories.

After the data have been taken, the time variation of the complex gains (amplitude and phase) has to be calibrated for the two data sets separately, since there is no correlation between the gain variations at the two sites. It is not necessary that both arrays use the same objects, usually quasars, as phase and amplitude calibrators. For the absolute calibration of the flux, however, it can be advantageous to include the same secondary calibrator (a bright quasar), the flux of which is derived from contemporaneous observations of a primary calibrator (planet), preferably with both arrays. This ensures that there are no offsets in flux calibration between the arrays. If the arrays have a different response to spatial scales, such flux offset would affect the resulting combined image.

Before the calibrated data sets can be combined, they need to be converted to a common data format (if not already the same). The OVRO data are usually exported in FITS format, while BIMA data are written in a format peculiar to the MIRIAD software package. The MIRIAD task FITS can convert FITS files to MIRIAD files. To successfully convert OVRO data using this task, use "options=varwt" so that the weights of the visibility data written by MMA (the OVRO data calibration package) are properly interpreted as the reciprocal of the noise variance. Further processing in the MIRIAD package does not require any additional conversions; many MIRIAD tasks accept input data sets that, for example, have different spectral gridding as do our OVRO and BIMA data.

Figure 3 illustrates the data combination procedure. To test our combination procedure, this figure also shows the results of combining data with a relative spatial offset; this results in an image with two "sources," each with approximately $50 \%$ of the flux, because in our observations, the sensitivities of the OVRO and BIMA data were about equal.

\section{REFERENCES}

A'Hearn, M. F., Millis, R. L., Schleicher, D. G., Osip, D. J., \& Birch, P. V. 1995, Icarus, 118, 223

Altenhoff, W. J., Bertoldi, F., Menten, K. M., Sievers, A., Thum, C., \& Kreysa, E. 2002, A\&A, 391, 353

Biver, N., et al. 1999, AJ, 118, 1850

Blake, G. A., Qi, C., Hogerheijde, M. R., Gurwell, M. A., \& Muhleman, D. O. 1999, Nature, 398, 213

Bockelée-Morvan, D., Crovisier, J., Baudry, A., Despois, D., Perault, M., Irvine, W. M., Schloerb, F. P., \& Swade, D. 1984, A\&A, 141, 411

Bockelée-Morvan, D., et al. 2001, Science, 292, 1339

Bonev, T., Jockers, K., Petrova, E., Delva, M., Borisov, G., \& Ivanova, A. 2002, Icarus, 160, 419

Cochran, A. L., \& Cochran, W. D. 2001, Icarus, 154, 381

Crovisier, J. 1987, A\&AS, 68, 223

Desvoivres, E., Klinger, J., Levasseur-Regourd, A. C., Lecacheux, J., Jorda, L., Enzian, A., Colas, F., Frappa, E., \& Laques, P. 1999, MNRAS, 303, 826

Durig, D., Ticha, J., \& Tichy, M. 1999, IAU Circ., 7267, 1

Farnham, T. L., Schleicher, D. G., Woodney, L. M., Birch, P. V., Eberhardy, C. A., \& Levy, L. 2001, Science, 292, 1348

Irvine, W. M., \& Bergin, E. A. 2000, in IAU Symp. 197, Astrochemistry: From Molecular Clouds to Planetary Systems, ed. Y. C. Minh \& E. F. van Dishoeck (San Francisco: ASP), 447

Irvine, W. M., Schloerb, F. P., Crovisier, J., Fegley, B., \& Mumma, M. J. 2000, in Protostars and Planets IV, ed. V. G. Mannings, A. P. Boss, \& S. S. Russell (Tucson: Univ. Arizona Press), 1159
Jockers, K., Bonev, T., Delva, M., Kiselev, N., \& Petrova, E. 2001, in Astronomische Gesellschaft Meeting Abstracts, 18, P08

Kidger, M. 2000, IAU Circ., 7467, 2 2002, Earth Moon Planets, 90, 157

Lisse, C. M., Christian, D. J., Dennerl, K., Meech, K. J., Petre, R., Weaver, H. A., \& Wolk, S. J. 2001, Science, 292, 1343

Magee-Sauer, K., Mumma, M. J., DiSanti, M. A., \& Dello Russo, N. 2001, BAAS, 33, 1076

Mäkinen, J. T. T., Bertaux, J., Combi, M. R., \& Quémerais, E. 2001, Science, 292,1326

Mumma, M. J., Weissman, P. R., \& Stern, S. A. 1993, in Protostars and Planets III, ed. E. H. Levy \& J. I. Lunine (Tucson: Univ. Arizona Press), 1177

Mumma, M. J., et al. 2001, Science, 292, 1334

Rodríguez, J., Sánchez, S., García, A., Blasco, M., \& Villalonga, M. A. 2002, Earth Moon Planets, 90, 185

Sault, R. J., Teuben, P. J., \& Wright, M. C. H. 1995, in ASP Conf. Ser. 77, Astronomical Data Analysis Software and Systems IV, ed. R. A. Shaw, H. E. Payne, \& J. J. E. Hayes (San Francisco: ASP), 433

Schulz, R., \& Stüwe, J. A. 2002, Earth Moon Planets, 90, 195

Scoville, N. Z., Carlstrom, J. E., Chandler, C. J., Phillips, J. A., Scott, S. L., Tilanus, R. P. J., \& Wang, Z. 1993, PASP, 105, 1482

Tozzi, G. P., \& Licandro, J. 2002, Icarus, 157, 187

Weaver, H. A., et al. 2001, Science, 292, 1329 